\title{
Caractéristiques de l'évolution spatio-temporelle de Pectinophora gossypiella Saunders (Lepidoptera : Gelechiidae) dans les zones de production cotonnière de Côte d'Ivoire : Implications pour une stratégie de gestion optimale de la résistance aux pyréthrinoïdes
}

\section{Germain Elisabeth Cynthia Ochou}

Université Nangui Abrogoua (UNA)

Laboratoire de Cytologie et de Biologie Abidjan, Côte d'Ivoire.

Koffi Christophe Kobenan

Université Félix Houphouët-Boigny (UFHB)

Laboratoire de Physiologie Végétale, Abidjan, Côte d'Ivoire.

Pitou Woklin Euloge Kone

Roland Didi

Université Nangui Abrogoua (UNA)

Laboratoire de Cytologie et de Biologie, Abidjan, Côte d'Ivoire.

\section{Malanno Kouakou}

Kouadio Kra Norbert Bini

Centre National de Recherche Agronomique (CNRA)

Laboratoire d'Entomologie Agricole, Bouaké, Côte d'Ivoire.

\section{Dagnogo Mamadou}

Université Nangui Abrogoua (UNA)

Laboratoire de Cytologie et de Biologie, Abidjan, Côte d'Ivoire.

\section{Acka Emmanuel Dick}

Université Félix Houphouët-Boigny (UFHB)/

Laboratoire de Physiologie Végétale, Abidjan, Côte d'Ivoire.

\section{Ochou Germain Ochou}

Centre National de Recherche Agronomique (CNRA)/

Laboratoire d'Entomologie, Bouaké, Côte d'Ivoire.

Doi: 10.19044/esj.2018.v14n21p217 URL:http://dx.doi.org/10.19044/esj.2018.v14n21p217

Abstract

In order to update knowledge on the spatio-temporal evolution of Pectinophora gossypiella, the geographical areas and the seasons of predilection of adult and larval populations have been studied in the last six years from 2012 to 2017 . The study showed that adult populations are more 
abundant in the cotton production area of the South compared to the North. They have a very low activity during the intercropping season while the most important outbreaks occurred in October-December. The monitoring of larval populations carried out on farm scale showed that infestations started on cotton early August and increased until November, which leads to a strong relationship between the evolution of adult populations, larval infestations and cotton plant phenology. Late sowings D5 and D6 exhibited higher levels of infestation (0.50 to 0.80 larvae/100 bolls) as compared to early sowing (0.21-0.31 larvae/100 bolls). Annual variations of pest infestation levels increased from years to years, fluctuating from 0.14 to 0.95 larvae/100 bolls over the study period. Mapping of the geographic distribution of infestation showed more severe pest pressure in the southern cotton areas, mostly in surrounding areas of Bouaké, Bouaflé, Séguéla and Boron $(18,90 ; 5,33 ; 1,52$; 1,06 larvae/100 bolls respectively). With regard to the emerging status of $P$. gossypiella over recent years, the current pyrethroid resistance management strategy deserves to be improved on the basis of the pest geographic and seasonal profiles.

Keywords: Pectinophora gossypiella, adult populations, larval infestations, geographic distribution, cotton.

\section{Resume}

Dans le but d'actualiser les connaissances sur l'évolution spatiotemporelle de Pectinophora gossypiella, l'aire géographique et la période de prédilection des populations adultes et larvaires ont été étudiées au cours des six dernières années de 2012 à 2017. L'étude a montré que les populations adultes sont plus abondantes dans la zone de production cotonnière du Sud par rapport à celle du Nord. Elles ont une très faible activité pendant l'intersaison alors que les pullulations les plus importants débutent du 22 Octobre au 17 Décembre. Le suivi des infestations larvaires sur un réseau de parcelles paysannes a montré que les infestations surviennent sur le cotonnier dès le début d'Août et s'accroissent jusqu'en Novembre. Ce qui induit une forte relation entre l'évolution des populations adultes, les infestations larvaires et la phénologie du cotonnier. Les semis tardifs D5 et D6 ont présenté des niveaux d'infestation plus importants $(0,50$ à 0,80 chenille/ 100 capsules) contre 0,21-0,31 chenilles/100 capsules pour les semis précoces. Les variations annuelles des niveaux moyens d'infestations ont augmenté d'années en années de 0,14 à 0,95 chenille/ 100 capsules sur la période d'étude. La cartographie de la distribution géographique des infestations a montré que la pression du ravageur est plus prononcée dans les zones cotonnières sud, particulièrement dans les localités de Bouaké, Bouaflé, Séguéla et Boron $(18,90 ; 5,33 ; 1,52 ; 1,06$ chenille/100 capsules 
respectivement). Au regard de l'aire géographique de prédilection de $P$. gossypiella et de l'évolution remarquable des infestations larvaires au cours des dernières années, l'actuelle stratégie de protection mérite d'être améliorée.

Mots clés : Pectinophora gossypiella, populations adultes, infestations larvaires, aire géographique, cotonnier.

\section{Introduction}

Le ver rose Pectinophora gossypiella Saunders fait parti du complexe des chenilles carpophages endocarpiques du cotonnier. Ce ravageur s'attaque aux organes fructifères et son développement a lieu entièrement à l'intérieur du fruit jusqu'à son dernier stade (Vaissayre et Cauquil, 2000 ; Lykouressis et al., 2005). C'est au cours de la récolte qu'il est constaté que des capsules apparemment saines sont détériorées de l'intérieur par les larves de P.gossypiella.

En Afrique sub-sahérienne, pour assurer le contrôle des ravageurs, la lutte chimique demeure la méthode la plus utilisée par les producteurs de coton. Néanmoins l'utilisation massive en Côte d'Ivoire depuis plusieurs années de certains produits insecticides, notamment les pyréthrinoïdes pour contrôler les Lépidoptères du cotonnier s'est traduite par une sélection d'individus résistants chez des ravageurs comme Helicoverpa armigera (Martin et al., 2000). Il s'est donc bâti autour de ce ravageur, à partir de 1998, une stratégie de gestion et de prévention de la résistance aux pyréthrinoïdes à travers des programmes «fenêtres ». Ce programme adopté en Côte d'Ivoire a consisté à réduire l'utilisation de produits à base de pyréthrinoïdes au profit de produits alternatifs (benzoylurées, diamides, organophosphorés, avermectines, etc) (Ochou et Martin, 2002 ; Doffou et al., 2011 ; Djihinto et al., 2016). Il a eu le succès escompté auprès des paysans en ayant exercé un bon contrôle sur les populations de Helicoverpa armigera.

Toutefois, au cours de ces dernières années d'importants changements ont été observés dans le faciès parasitaire du cotonnier. Cette situation suscite des interrogations, notamment sur la sensibilité de certains ravageurs du complexe parasitaire du cotonnier aux insecticides utilisés. Doffou (2012) a détecté une perte de sensibilité chez deux chenilles endocarpiques du cotonnier Thaumatotibia leucotreta et $P$. gossypiella au cours de ces travaux menés en Côte d'Ivoire.

Toutes ces préoccupations ont amené à remettre en cause les stratégies de protection dont l'efficacité repose en grande partie sur une bonne maîtrise de la distribution spatio-temporelle et des niveaux de sensibilité aux insecticides utilisés en culture cotonnière. 
Cette étude basée sur un suivi des populations adultes et larvaires de $P$. gossypiella, en culture cotonnière en Côte d'Ivoire, a pour objectif d'actualiser les connaissances sur $P$. gossypiella en vue d'élaborer une option stratégique de gestion optimale du ravageur, en tenant compte des nouvelles contraintes et du faciès parasitaire actuel.

\section{Matériel et méthodes}

\section{Insecte ravageur cible}

La présente étude a concerné les adultes et les larves du Lépidoptère $P$. gossypiella de la famille des Gelechiidae. Les populations adultes naturelles mâles de l'insecte, volent surtout la nuit. Les populations larvaires principalement carpophages endocarpiques vivant à l'intérieur des fleurs ou se réfugiant à l'intérieur des capsules vertes, ont été aussi suivies durant toute la période de floraison et fructification du cotonnier.

\section{Sites d'étude}

Le site d'étude a concerné l'ensemble de la zone de culture cotonnière de la Côte d'Ivoire (Figure 1). Elle s'étend du Nord au Centre c'est-à-dire en région des savanes, précisément entre $6^{\circ}$ et $10^{\circ} 50$ de latitude Nord, $4^{\circ}$ et $8^{\circ}$ de longitude Ouest. Cette zone d'étude comprend la station de recherche cotonnière basée à Bouaké et l'ensemble des zones administratives de production cotonnière des sociétés cotonnières. Ces localités sont réparties au regard de la pluviométrie en deux grandes régions par rapport au $9^{\text {ème }}$ parallèle, à savoir : la région Nord pour toutes les localités cotonnières au-dessus du 9 ème parallèle, avec une seule saison de pluie ; la région Sud pour toutes les localités cotonnières en dessous du $9^{\text {ème }}$ parallèle, avec deux saisons de pluie.

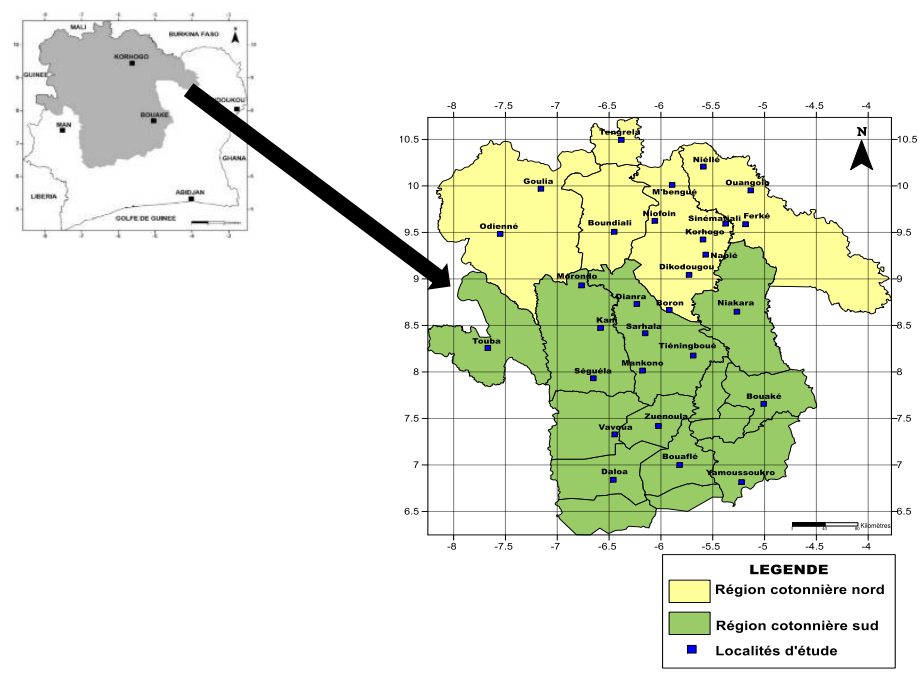

Figure 1 : Zone de production cotonnière de Côte d'Ivoire 


\section{Suivi des populations naturelles adultes}

Le suivi des populations adultes a eu lieu à la station de recherche sur le coton, basée à Bouaké, sur toute la période de l'année de Janvier à Décembre, pendant cinq années consécutives allant de 2013 à 2017. Le piège à phéromone a été implanté en bordure du bloc de champs de cotonniers. Il est de confection locale, représenté par une bouteille d'eau minérale vide de type «bouteille d'eau minérale AWA »d'une contenance de 1,5 litre. Trois ouvertures de $2 \mathrm{~cm} \mathrm{x} 4 \mathrm{~cm}$ ont été fait sur le tiers supérieur de la bouteille en plastique. La moitié supérieure de la bouteille a été peinte en noir. Le fond de la bouteille a été rempli d'eau jusqu'à la $4{ }^{\text {ème }}$ rainure (environ $10 \mathrm{~cm}$ ) en prenant soin d'y ajouter un peu de détergent liquide. La charge de phéromone a été suspendue par un fil en fer à l'intérieur de la bouteille, à travers le bouchon de la fermeture de façon à la réajuster au niveau des orifices latéraux. La bouteille d'eau a été accrochée à un pieu en bois d'environ $2 \mathrm{~m}$ du sol. La direction du vent a été prise en compte lors de l'emplacement du piège. La charge de phéromone suspendue à l'intérieur de la bouteille a été changée toutes les quatre (04) semaines.

La phéromone utilisée dans la présente étude pour la surveillance des populations naturelles de $P$. gossypiella a été fournie sous la forme de capsule en polyéthylène en sachet hermétique individuel par la société Russell IPM Ltd, sous le code PH-702-1RR. La capsule peut libérer progressivement pendant quatre à cinq semaines la charge de phéromone (1-2 mg/capsule) dans l'atmosphère avec une intensité proche de celle de la femelle naturelle.

Le comptage des individus adultes mâles capturés par le piège de surveillance a été effectué 3 fois par semaine durant les campagnes agricoles et les intercampagnes. L'abondance des captures hebdomadaires réalisées a permis d'établir la dynamique saisonnière des populations adultes mâles.

\section{Suivi des infestations larvaires}

Le suivi des infestations larvaires a eu lieu en milieu paysan dans les zones cotonnières pendant six campagnes agricoles consécutives de 2012 à 2017. Pendant chaque campagne agricole, 10 parcelles paysannes ont été sélectionnées par zone, réparties de façon proportionnelle sur les différentes décades de semis les plus représentatives de la zone de production. Les parcelles ont été choisies chez des producteurs appliquant le programme de lutte sur calendrier. Le dispositif de suivi parasitaire, dépendant des organisations administratives et techniques des sociétés cotonnières, a donc impliqué des parcelles paysannes de 0,25 ha dont le nombre total a varié selon les années entre 360 et 500.

Dans chaque parcelle paysanne, des Analyses Sanitaires de Capsules Vertes (ASCV) ont été effectuées sur des capsules vertes de même âge (diamètre supérieur à $2 \mathrm{~cm}$ ) à raison de 50 capsules par parcelle. Elles ont été 
prélevées au milieu du plant et proches de la tige principale et analysées aux $80^{\text {ème }}, 87^{\text {ème }}, 94^{\text {ème }}, 101^{\text {ème }}$ et $108^{\text {ème }}$ jours après levée (JAL). Lors du dépouillement, les chenilles de $P$. gossypiella ont été dénombrées parmi les autres espèces carpophages rencontrées. Des niveaux moyens d'infestations larvaires ont été établis par zone, ce qui a permis de déterminer leurs variations annuelles, saisonnières et géographiques.

\section{Traitement et analyse statistique des données}

Les données parasitaires obtenues ont été saisies à l'aide du logiciel Excel 2013 en vue de l'établissement de la dynamique saisonnière des populations adultes, des variations annuelles et saisonnières des infestations larvaires ainsi que les relations entre les populations adultes et larvaires, et les niveaux d'infestations larvaires et les décades de semis. Des barres d'erreur standard ont été matérialisées sur certains graphiques à partir des écart-types calculés. Le logiciel de cartographie Surfer version 11.5.1069 qui se prête bien à la spatialisation de données a permis d'établir les cartes de distribution géographique des infestations parasitaires. Le logiciel SPSS version 20 a permis de faire l'analyse de variance. En cas de différence significative (seuil de 0,05 ), le test de Duncan a permis de dégager les différents groupes ou sousensembles homogènes qui sont représentés par des lettres alphabétiques $a, b$, c, etc. Ainsi les moyennes suivies des mêmes lettres appartiennent au même groupe.

\section{Résultats}

\section{Dynamique saisonnière des populations naturelles de $\boldsymbol{P}$. gossypiella}

Dans la zone cotonnière, les figures 2 et 3 montre les captures de papillons de $P$. gossypiella qui diffèrent d'une région à l'autre sur une période allant de 2013 à 2017.

En effet, l'activité des adultes a été plus marquée dans la localité de Bouaké (Figure 2) que dans les autres régions cotonnières, Nambingué, Korhogo, Boundiali, Séguéla, et Niakara (Figures 3 a, b, c, d, e, f). A Bouaké, il a été noté la présence de deux pics importants au cours des campagnes cotonnières, ayant lieu à la $43^{\text {ème }}$ semaine $\left(22\right.$ Octobre) et à la $51^{\text {ème }}$ semaine (17 Décembre). Les populations adultes ont été présentes au cours de toute la période d'observation mais les deux pics atteints ont été respectivement en :

2014: de 457 papillons capturés/semaine et 462 papillons
capturés/semaine;
2015: de 464 papillons capturés/semaine et 860 papillons
capturés/semaine;
2017: de 521 papillons capturés/semaine et 425 papillons
capturés/semaine.




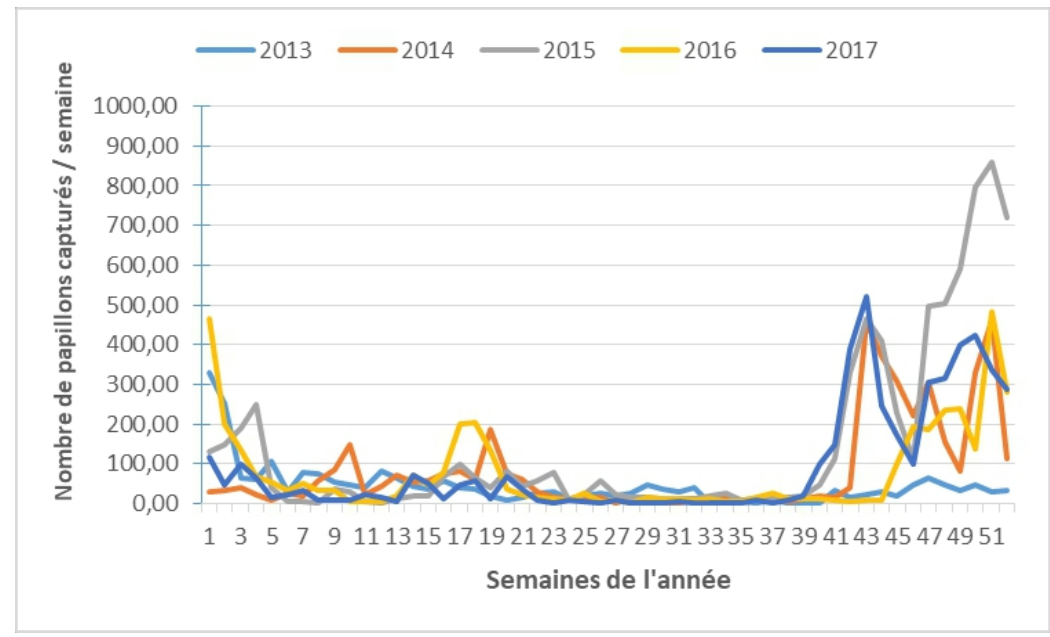

Figure 2 : Fluctuations hebdomadaires des captures de $P$. gossypiella à la station de recherche à Bouaké (zone cotonnière sud) de 2013 à 2017

Toutefois, les captures ont été peu abondantes en 2013 traduisant une faible présence de $P$. gossypiella au regard des autres campagnes cotonnière (64 papillons capturés/semaine et 48 papillons capturés/semaine). Les populations adultes de $P$. gossypiella ont été quasiment absentes ou peu abondantes dans certaines régions cotonnières au cours de la période de 2013 à 2017.

A Nambingué, les captures ont été plus importantes en 2015 (20 Août avec 13 papillons capturés/semaine) et peu abondantes en 2017 (30 Juillet avec 3 papillons capturés/semaine). Pour les années 2013, 2014 et 2016, les captures ont été quasi inexistantes (Figure 3a).

Concernant Korhogo, pour les années 2014, 2015, 2016 et 2017, les pics les plus importants ont été respectivement de 45, 22, 12 et 40 papillons capturés/semaine aux dates suivantes: 03 Décembre, 26 novembre, 12 Novembre et 19 Novembre. La présence des papillons a été plus marquée en 2014. (Figure 3b).

A Boundiali, les populations d'adultes ont été observées de 2014 à 2017 excepté 2015 où les captures des adultes ont été peu abondantes. Le pic le plus important en 2014, est apparu le 26 Novembre avec 376 papillons capturés/semaine, et en 2016, le 01 Octobre, avec 104 papillons capturés/semaine. En 2017, le pic le plus important a été observé le 15 Octobre avec 73 papillons capturés/semaine (Figure 3c).

Dans la localité de Séguéla, les années 2014, 2015 et 2016 ont présenté leurs pics les plus importants à la même date d'observation c'est-à-dire le 19 Novembre avec des valeurs respectives de 247,77 et 48 papillons 
capturés/semaine. En 2017, 225 papillons capturés/semaine, le 06 Août, ont représenté le pic le plus important de l'année (Figure 3d).

Pour la localité de Niakara, seules les années 2015 et 2016 ont montré des captures importantes avec 757 papillons capturés/semaine le 19 Novembre et de 44 papillons capturés/semaine le 03 Septembre.

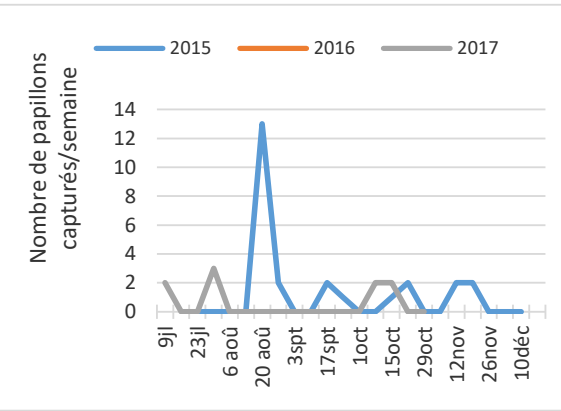

Figure 3a : Nambingué (zone extrême nord)

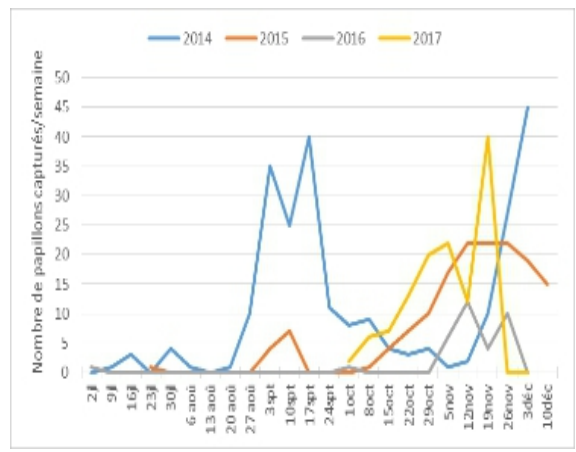

Figure $3 b$ : Korhogo (zone nord)

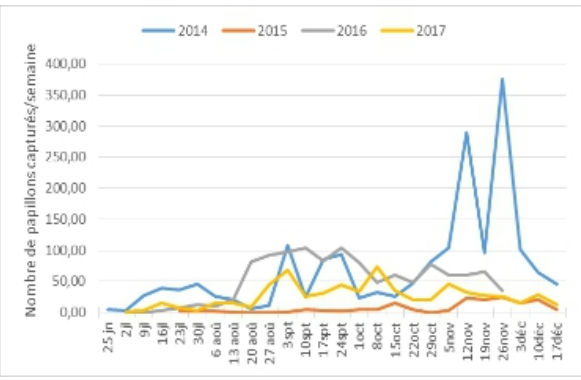

Figure 3c : Boundiali (zone nord-ouest)

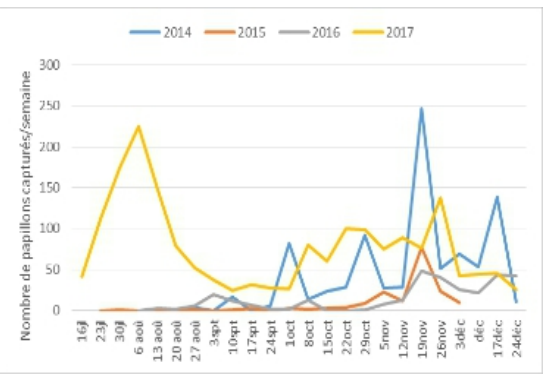

Figure 3d : Séguéla (zone sud-ouest)

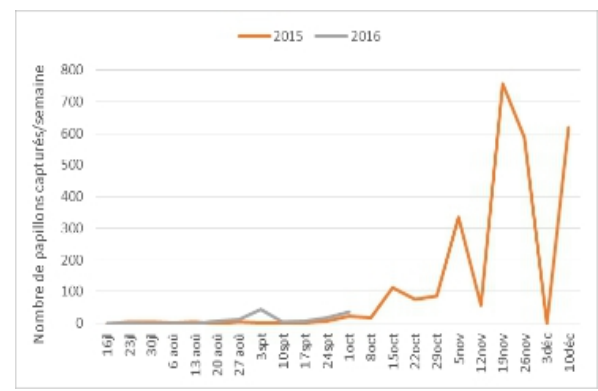

Figure $3 \mathrm{e}$ : Niakara (zone sud centre)

Figure 3 : Dynamique saisonnière des populations adultes de P. gossypiella dans différentes régions cotonnières 
Variations annuelles d'abondance des populations adultes de $P$. gossypiella à la station de recherche à Bouaké (zone cotonnière sud) de 2013 à 2017

L'abondance des populations adultes de $P$. gossypiella a varié selon les années dans la localité de Bouaké (Figure 4). De 2013 à 2017, les niveaux de populations adultes capturées ont été respectivement de 44,31 papillons capturés/semaine ; 83,35 papillons capturés/semaine; 141,92 papillons capturés/semaine ; 77,17 papillons capturés/semaine et 92,34 papillons capturés/semaine. L'analyse statistique a révélé une différence significative entre les niveaux de papillons capturés par semaine au cours des différentes années. Deux groupes homogènes ont été observés et le taux moyen de capture le plus important a été obtenu en 2015 (141,92 papillons capturés/semaine) contre un taux en 2013 de 44,31 papillons capturés/semaine.

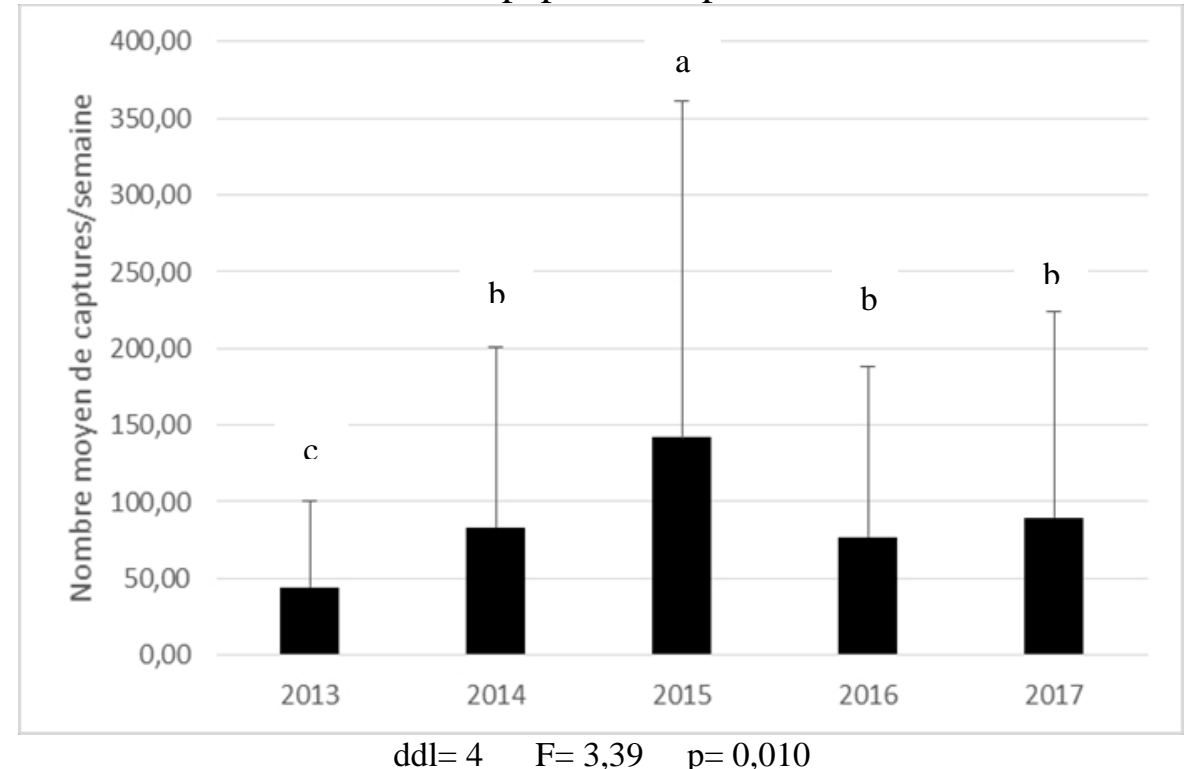

Figure 4 : Evolution des niveaux moyens de captures hebdomadaires des populations adultes de $P$. gossypiella à Bouaké au cours des années de 2013 à 2017

\section{Variations géographiques d'abondance des populations adultes}

L'activité des populations adultes dans la zone cotonnière est illustrée par la figure 5. Elle dénote ainsi que la présence d'adultes été plus marquée dans la localité de Bouaké (175,34 papillons capturés/semaine) que dans celles de Nambingué (0,86 papillons capturés/semaine), suivie de Korhogo (7,66 papillons capturés/semaine), Boundiali (39,23 papillons capturés/semaine), Séguéla (37,32 papillons capturés/semaine) et Niakara (66,69 papillons capturés/semaine). L'analyse statistique a révélé une différence significative entre les niveaux moyens des populations adultes capturés des différentes régions de la zone cotonnière (Figure 5). 


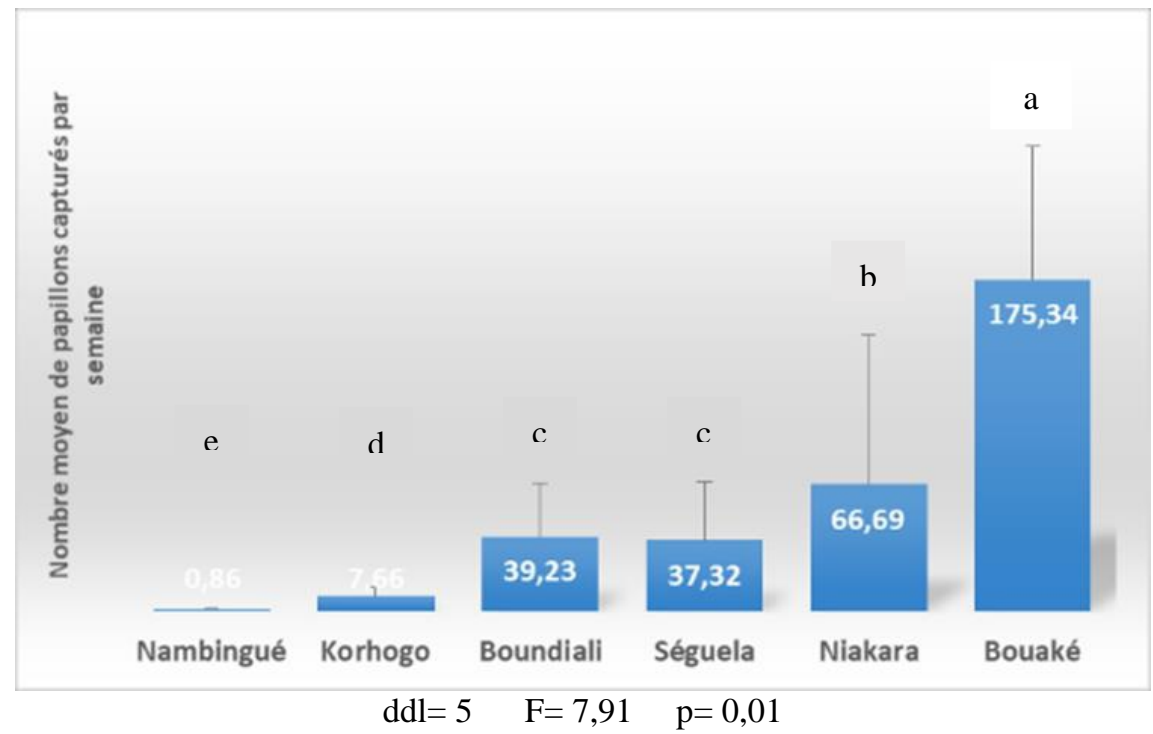

Figure 5 : Niveaux moyens des captures hebdomadaires de populations adultes de $P$. gossypiella réalisées dans les différentes régions au cours des campagnes agricoles de 2014 à 2017

\section{Distribution saisonnière des infestations larvaires de $\boldsymbol{P}$. gossypiella}

La figure 6 présente l'évolution saisonnière des infestations larvaires de P.gosssypiella de 2012 à 2017, ainsi que la courbe représentant la tendance générale de l'évolution. Les infestations larvaires de $P$. gossypiella au cours des six années d'observations, ont débuté à partir du mois de Juillet, puis ont évolué de manière progressive jusqu'à atteindre des pics plus importants au mois de Novembre. La tendance générale de la courbe de l'évolution saisonnière traduit une croissance des infestations larvaires de $P$. gossypiella à partir de fin Août jusqu'en Novembre. Les pics les plus importants observés ont été en 2012 de 0,75 chenille pour 100 capsules qui a eu lieu le 4 octobre, et de 0,24 chenille pour 100 capsules ( 27 octobre) en 2013. En 2014 et 2015, les pics ont été respectivement de 0,39 et 1,31 chenille pour 100 capsules observés le 20 Septembre et le 02 Novembre. Les deux dernières années 2016 et 2017, un pic de 0,9 (08 novembre) et un autre de 0,40 chenille pour 100 capsules (01 novembre) ont été obtenus. 


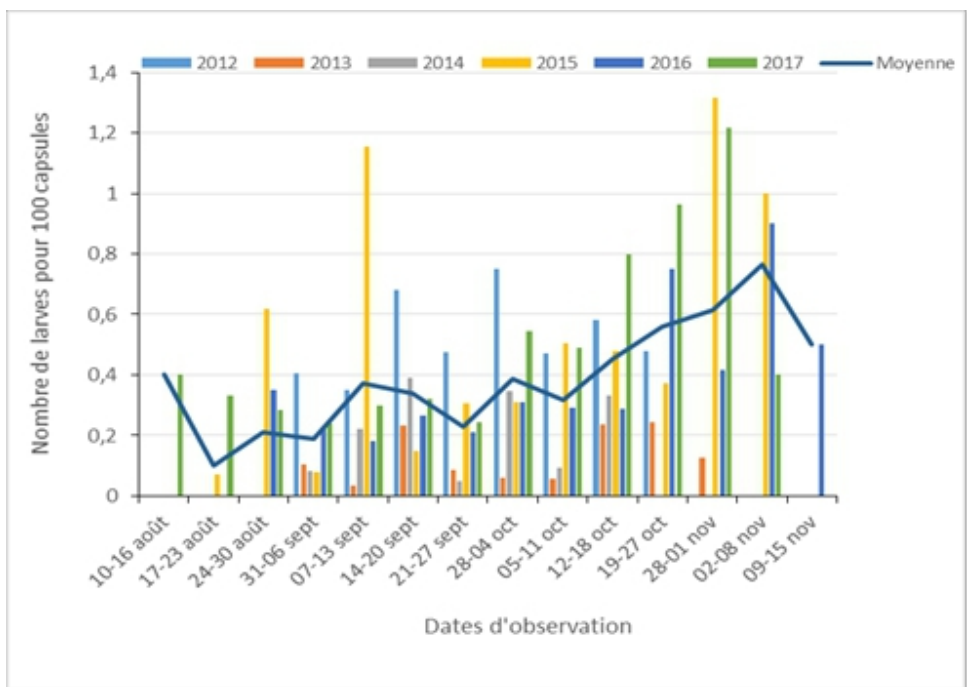

Figure 6 : Evolution saisonnière des infestations larvaires de $P$. gossypiella sur capsules vertes de 2012 à 2017

\section{Evolution des infestations larvaires de $\boldsymbol{P}$. gossypiella selon les décades de semis}

Au cours de cette période 2012 - 2015, chez P. gossypiella, les dates de semis ont présenté une incidence variable sur les niveaux d'infestations. Les infestations observées en fonction des décades de semis ont varié de 0,07 à 0,22 chenille/100 capsules. De la décade 1 à la décade 6 , les niveaux moyen d'infestations ont été respectivement de 0,$28 ; 0,33 ; 0,21 ; 0,31 ; 0,50$ et 0,80 chenille/100 capsules (Figure 7). L'analyse statistique a révélé une différence significative entre les niveaux d'infestations par décades de semis. Deux groupes homogènes ont été dégagés. Les semis tardifs (décades D5 et D6) ont été les plus infestés par $P$. gossypiella.

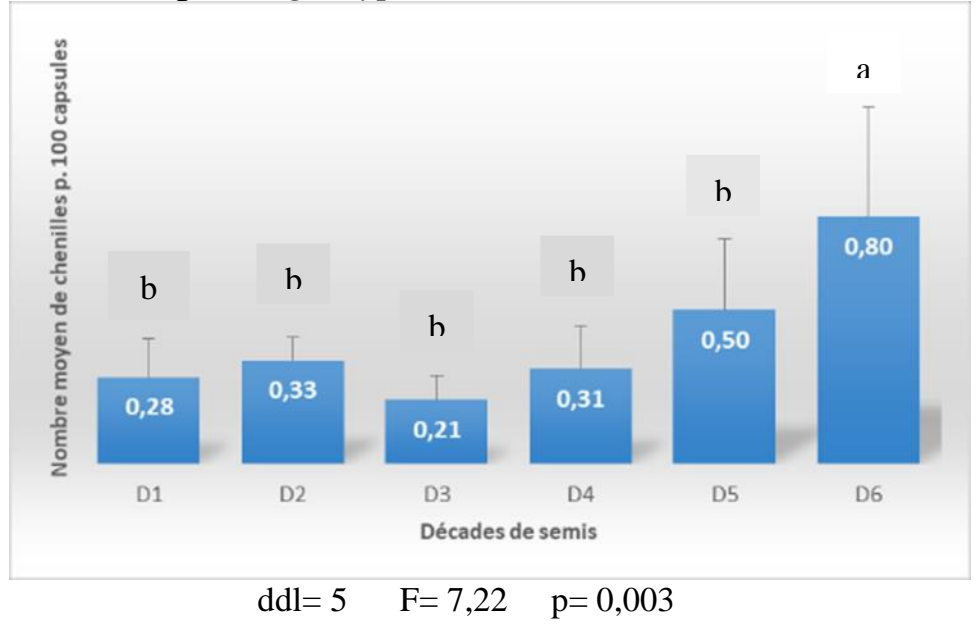

Figure 7 : Niveaux moyens d'infestation larvaires de $P$. gossypiella en fonction des décades de semis du coton. 


\section{Variations annuelles des infestations larvaires de $\boldsymbol{P}$. gossypiella}

Le niveau moyen des infestations larvaire de $P$. gossypiella a fluctué d'année en année, allant de 0,14 chenille/100 capsules à 0,95 chenille/100 capsules. Il est passé de 0,26 chenille/100 capsules en 2012 à 0,14 chenille/100 capsules en 2013, ensuite en 2014, il a été observé 0,21 chenille/100 capsules puis 0,29 chenille/100 capsules en 2015 . Les années 2016 et 2017 ont montré des niveaux respectifs de 0,45 et 0,95 chenille/100 capsules. Le niveau moyen général au cours de cette période a été de 0,94 chenille/ 100 capsules. Le niveau d'infestations larvaires le plus important a été observé en 2017. L'analyse statistique n'a révélé aucune différence significative entre les niveaux moyens d'infestations annuelles (Figure 8).

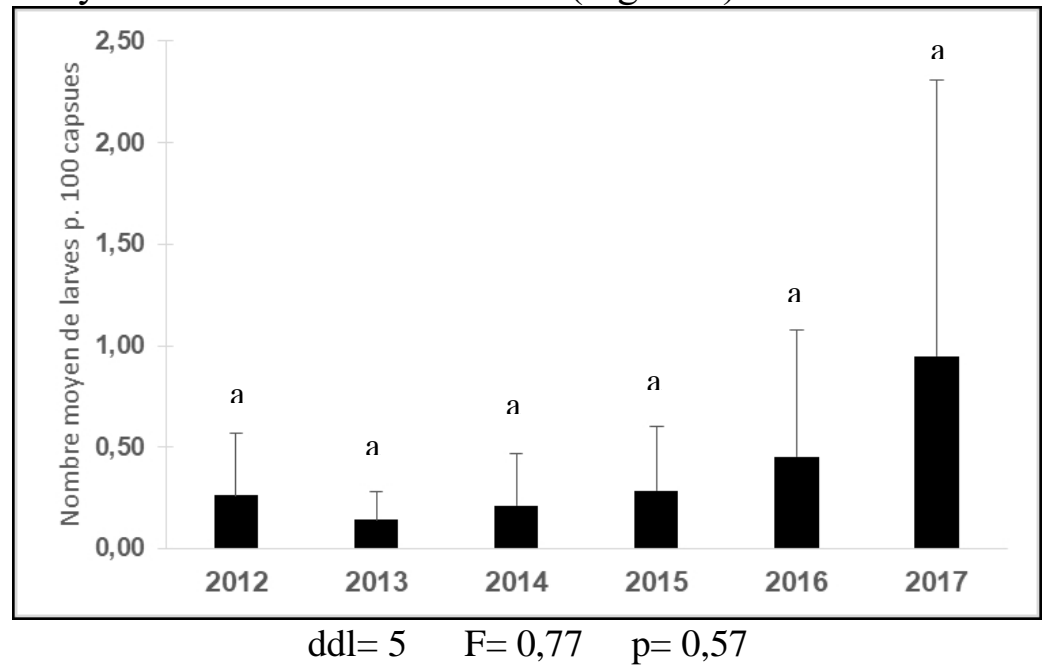

Figure 8 : Variations annuelles des niveaux moyens d'infestations larvaires de $P$.

gossypiella

\section{Evolution comparée entre populations larvaires et adultes de $P$. gossypiella}

La comparaison graphique de l'évolution des populations adultes et larvaires de $P$. gossypiella durant la période de 2012 à 2017 (Figure 9) a montré une évolution presqu'en phase des populations adultes et des niveaux d'infestations larvaires sur capsules. Le pic de croissance des populations adultes a lieu environ une semaine plus tôt par rapport au pic de croissance des larves (22 octobre vs 29 octobre). Une forte corrélation positive a été déterminée entre les populations adultes et les infestations larvaires de $P$. gossypiella. Environ $66 \%$ des variations des infestations larvaires de ce lépidoptère seraient donc liés aux pullulations des populations adultes. 


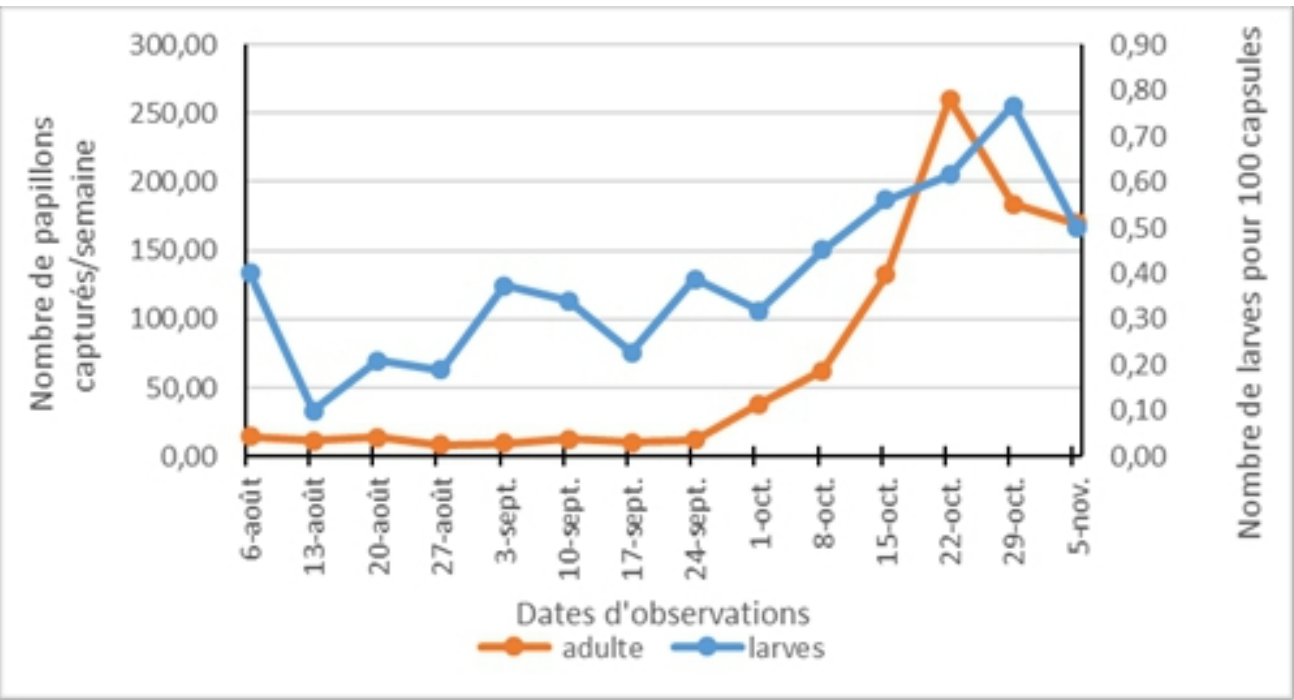

Figure 9 : Evolution comparée des larves et adultes de $P$. gossypiella durant la période de 2012 à 2017

\section{Distribution géographique des infestations larvaires de $\boldsymbol{P}$. gossypiella}

La distribution géographique des infestations larvaires sur le cotonnier a présenté un profil variable selon les périodes et les localités. La cartographie a montré au cours de la période allant de 2012 à 2015, trois épicentres localisés, dont deux dans la zone Sud et un dans la zone Nord (Figure 10a). De fortes infestations ont été observées dans les localités de Séguéla (1,52 chenille/100 capsules), M'Bengué (1,32 chenille/100 capsules) et Boron (1,06 chenille/100 capsules).

En 2015, P. gossypiella a été plus présent dans la zone Sud cotonnière. Les localités les plus attaquées ont été Séguéla (1,52 chenille/100 capsules), Vavoua (1,40 chenille/100 capsules) et Boron (1,12 chenille/100 capsules) (Figure 10b) ;

En 2016, les infestations ont été plus importantes dans la partie Sud, précisément dans la zone de Bouaflé (5,33 chenilles/ 100 capsules) et de Séguéla (1,92 chenilles p.100 capsules. Cependant, la zone de M'Bengué dans le Nord a également subi des attaques non négligeables (2,67 chenilles / 100 capsules) (Figure 10c) ;

En 2017, les infestations ont été plus accentuées dans la zone cotonnière Centre, Bouaké $(18,90$ chenilles/100 capsules et Sarhala $(3,18$ chenilles/100 capsules) (Figure 10d). 


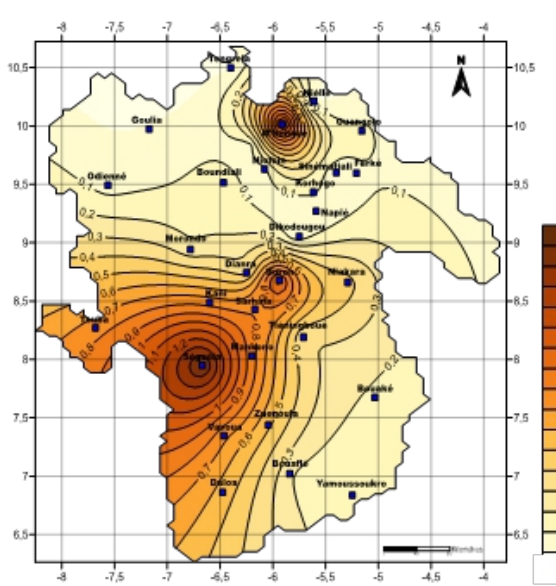

Figure 10a : Distribution géographique des niveaux d'infestations de $P$. gossypiella de 2012-2015 dans la zone cotonnière de Côte d'Ivoire

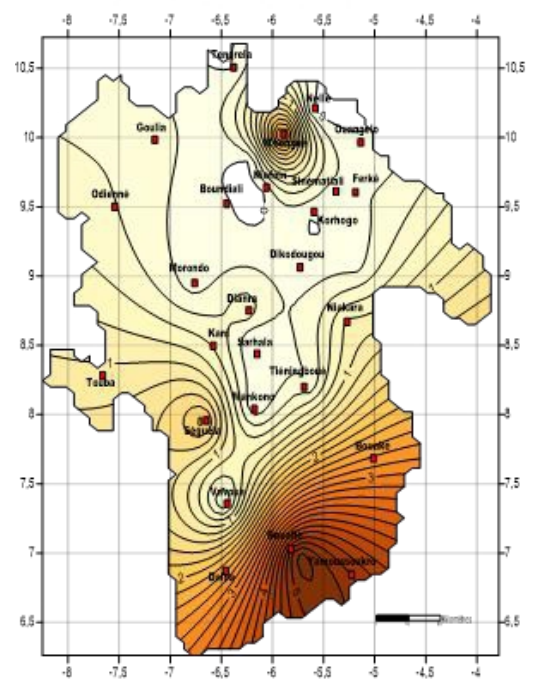

Figure 10c : Distribution géographique des infestations de $P$. gossypiella dans la zone cotonnière de Côte en 2016 d'Ivoire

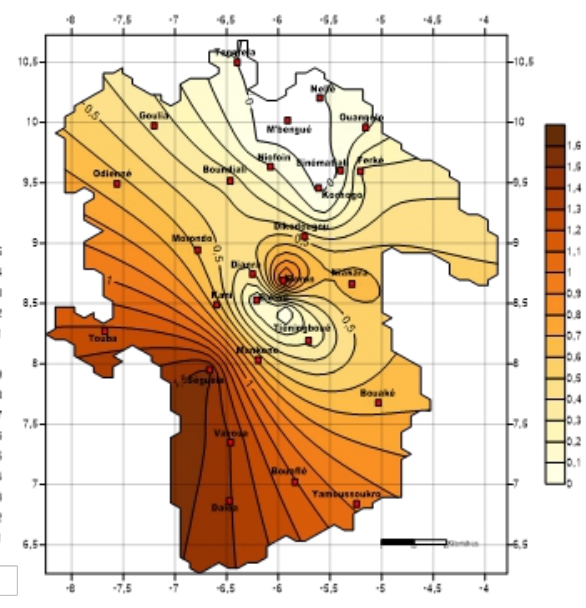

Figure 10b : Distribution géographique des infestations de $P$. gossypiella dans la zone cotonnière de Côte d'Ivoire en 2015

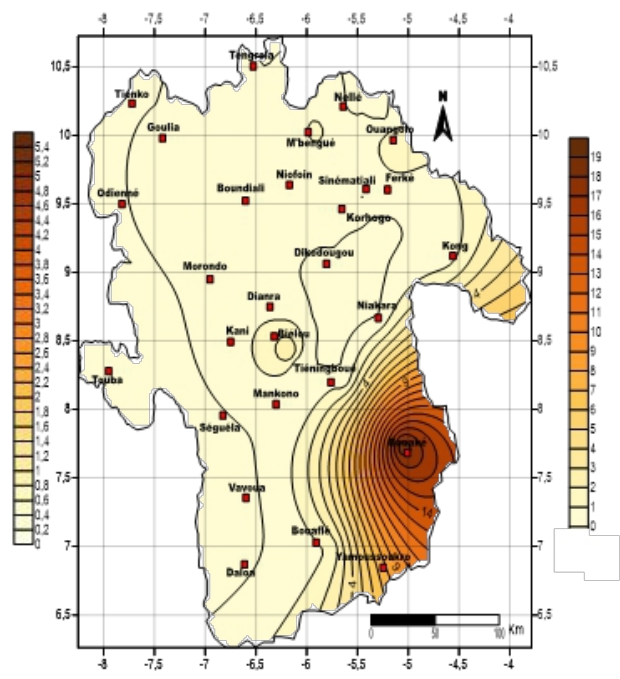

Figure 10d : Distribution géographique des infestations de $P$. gossypiella dans la zone cotonnière de Côte d'Ivoire en 2017

Figure 10 : Distribution géographique des infestations de $P$. gossypiella dans la zone cotonnière 


\section{Discussion}

\section{Prévision des risques d'infestations de $P$. gossypiella}

La forte corrélation observée entre les populations adultes et les infestations larvaires permet d'anticiper et d'appréhender mieux les périodes de fortes pullulations larvaires de $P$. gossypiella. Les différentes périodes de pullulations qui ont été déterminées chez les populations naturelles de $P$. gossypiella représentent un bon indicateur des niveaux d'infestations sur les parcelles cotonnières. Néanmoins, il est indispensable de comprendre profondément leur dynamique spatio-temporelle en réalisant des observations complémentaires (variétés cultivées et plantes hôtes environnantes, données pluviométriques journalières des postes les plus proches, relevés phénologiques, etc.). Les différentes périodes de pics et de baisse d'activité des papillons adultes pourraient être liées au stade phénologiques des cotonniers dans chaque localité comme il a été observé chez les altises en Côte d'Ivoire (Hala et al., 2006). Attique et al., 2004 ont aussi observer que l'abondance des populations évolue au fur et à mesure que la plante serait dans sa phase fructifère. La connaissance de tous les paramètres environnants et les données parasitaires permettraient d'élaborer une stratégie de gestion optimale (système d'alerte /avertissement) dans chaque grande zone de production. La prévision des risques d'infestations de ce lépidoptère carpophage du cotonnier est donc possible en Côte d'Ivoire.

\section{Aire géographique de prédilection des populations adultes et larvaires}

La cartographie de la distribution géographique des infestations larvaires de $P$. gossypiella a montré une distribution variable des niveaux de population par localité. La zone Sud cotonnière subit beaucoup plus la pression parasitaire du ravageur avec des localités fortement infestées et se révèle être favorable au développement de ce dernier. Les conditions climatiques et la disponibilité de plantes hôtes constitueraient ainsi l'attractivité de cette zone pour ce ravageur.

\section{Période critique de pullulations des populations adultes et larvaires}

La période critique des infestations de $P$. gossypiella débute en Août et peut s'étendre jusqu'en Octobre. Les pics de captures les plus importants ont été observés pour les localités de Bouaké, Niakara et Boundiali pratiquement à la même période (Août-Octobre-Novembre). La présence simultanée du ravageur dans les trois localités pourrait s'expliquer par la présence d'individus sédentaires qui contrairement aux migrateurs ont la possibilité d'entrer en diapause puis de reprendre leur développement lorsqu'ils sont en présence de conditions favorables (Unlu and Bilgic, 2004). Guttierrez et al. (2006) au cours de leurs travaux ont ainsi montré les capacités de $P$. gossypiella, en Arizona et en Californie, à faire face à certaines 
contraintes climatiques en entrant en diapause. Cependant, les papillons de $P$. gossypiella sont tout aussi capables de se déplacer comme l'ont observé Byers and Naranjo, (2014) dans le Nord-Ouest des Etats-Unis et dans certaines parties du Mexique. Toutefois, cette migration est surtout conditionnée par la présence de nourriture, de refuge et non par des variations climatiques.

Les différentes périodes de pics et de baisse d'activité des papillons adultes pourraient être en rapport avec le stade phénologique des cotonniers dans chaque localité. A ces dates, les parcelles peuvent présenter un environnement propice à l'apparition et à la multiplication de $P$. gossypiella dont les premiers pics coïncident avec la phase de formations des organes fructifères des cotonniers (Attique et al., 2004).

\section{Décades de semis favorables au développement des infestations larvaires}

Les infestations par décades de semis montrent que $P$. gossypiella est plus abondant sur les semis tardifs. L'abondance des ravageurs en fonction des décades de semis pourrait être liée à leur écologie. Clementine et al. (2005) ont montré que l'indisponibilité saisonnière des plantes pouvait amener les ravageurs à migrer sur des plantes hôtes secondaires en attendant le moment propice pour revenir. La date de semis est un facteur très important pour les agriculteurs et est généralement fonction des conditions climatiques de chaque région. Elle définit de ce fait le calendrier cultural d'une région. Jouer sur la date de semis permet de séparer la période de sensibilité de la plante hôte et la période d'abondance des ravageurs (Stoll, 2002).

\section{Définition d'une stratégie de gestion optimale}

Les niveaux d'infestation larvaires de P. gossypiella de 2012 à 2017 ont flucté au fil des années. Le programme phytosanitaire vulgarisé en Côte d'Ivoire semble avoir exercé un contrôle plus ou moins variable sur $P$. gossypiella.

Les niveaux d'infestation larvaires de 2012 à 2017 ont pratiquement doublé traduisant une croissance des populations du ravageur. Ces observations se justifieraient pour le ravageur d'une part, par son régime endocarpique qui lui permet d'éviter le contact avec les insecticides utilisés au cours des traitements; d'autre part, par une capacité de ce ravageur à métaboliser les doses d'insecticides reçues. Cette faculté à métaboliser les insecticides serait liée à une perte de sensibilité du ravageur aux insecticides utilisés comme l'a montré Doffou (2012) sur des souches de $P$. gossypiella provenant d'Abidjan (Côte d'Ivoire).

La stratégie de gestion de la résistance aux pyréthrinoïdes mis en place en culture cotonnière de Côte d'Ivoire, doit désormais prendre en compte $P$. gossypiella, surtout dans les zones de prédilection en recommandant à la 
première fenêtre non seulement des molécules alternatives aux pyréthrinoïdes mais aussi efficaces contre P. gossypiella (Figure 11).

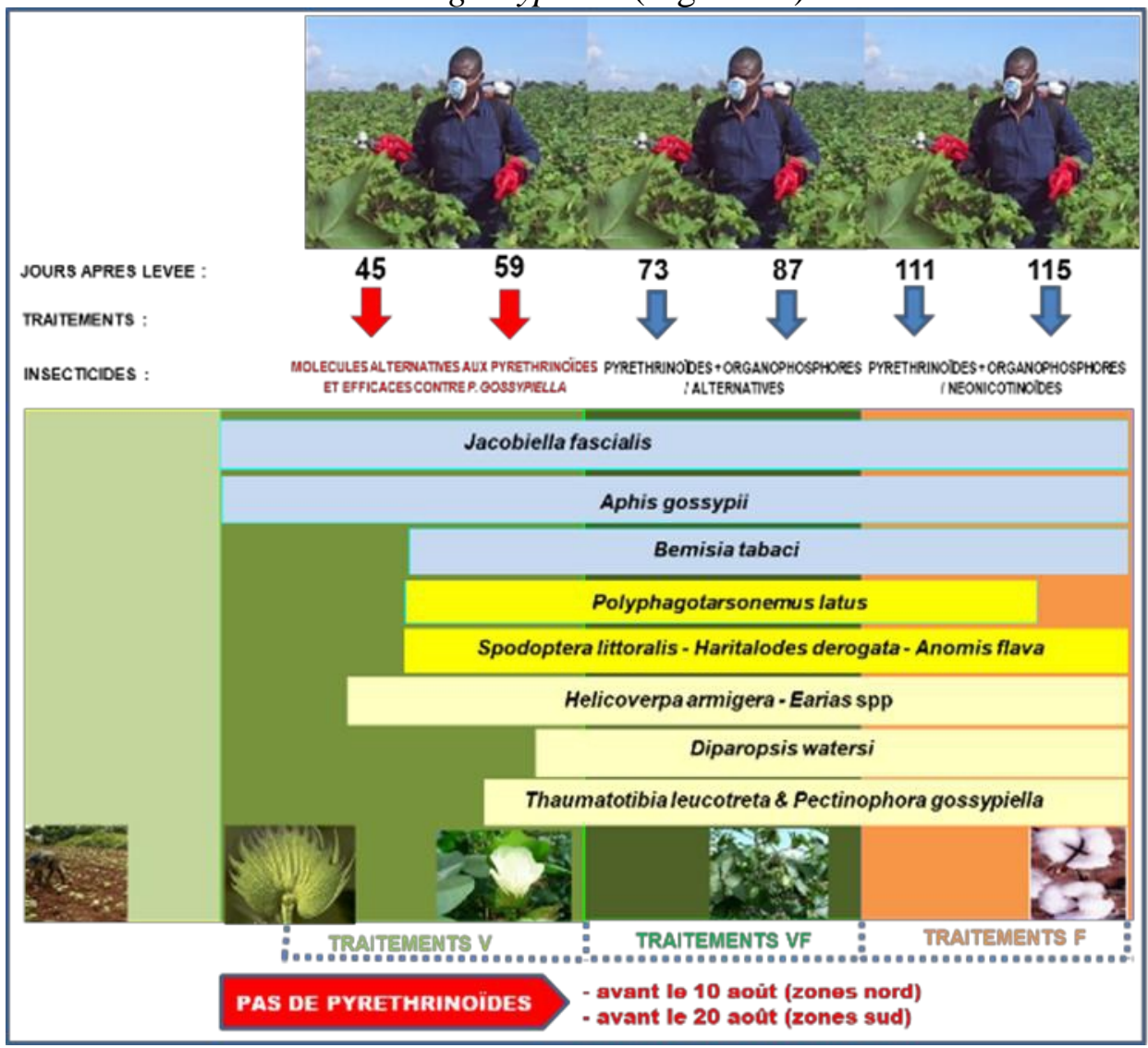

Figure 11 : Stratégie de gestion de la résistance aux pyréthrinoïdes prenant en compte $P$. gossypiella en culture cotonnière de Côte d'Ivoire.

\section{Conclusion}

L'étude de l'évolution spatio-temporelle du carpophage à régime endocarpique, $P$. gossypiella, a mis en évidence de 2012 à 2017 une croissance des infestations larvaires. La cartographie de la distribution géographique des niveaux d'infestation a révélé que la zone Sud cotonnière demeure la zone de prédilection du ravageur. La période critique des infestations maximales semble tardive chez $P$. gossypiella. La période de pullulation du ravageur est rendue favorable par la formation des fleurs, des capsules et des semis tardifs. Ces informations montrent clairement que la mise en place du programme de protection en vigueur a exercé un contrôle plus ou moins variable sur les populations du ravageur. Il serait donc important de poursuivre l'étude en tenant compte des paramètres écologiques et climatiques en vue d'élaborer une option stratégique de gestion optimale de $P$. gossypiella. 


\section{References :}

1. Attique, M.R., Ahmad, Z., Mohyuddin, A.I. \& Ahmad, M.M. (2004). Oviposition site preference of Pectinophora gossypiella (Lepidoptera: Gelechiidae) on cotton and its effects on boll development. Crop $\begin{array}{llll}\text { Protection } & 23 & \text { (4): } & \text { 287-292. }\end{array}$ https://doi.org/10.1016/j.cropro.2003.08.013

2. Byers, J.A. \& Naranjo, S.E. (2014). Detection and monitoring of pink bollworm moths and invasive insects using pheromone traps and encounter rate models J. Appl. Ecol. 51(4):1041-1049. DOI: $10.1111 / 1365-2664.12270$

3. Clementine, D., Antoine, S., Herve, B. \& Kouahou, F.B. (2005) Alternative host plants of Clavigralla tomentosicollis Stal. (Hemiptera:Coreidae), the pod sucking bug of cowpea in the Sahelian zone of Burkina Faso. $J$ Entomol 2:9-16

4. Djihinto, C.A., Affokpon, A., Dannon, E. \& Bonni, G. (2016). Le profenofos, un alternatif à l'endosulfan en culture cotonnière au Bénin. Int. J. Biol. Chem. Sci., 10(1): 175-183. DOI : http://dx.doi.org/10.4314/ijbcs.v10i1.12

5. Doffou, N.M. (2012). Impact de la gestion de la résistance aux pyréthrinoïdes sur les populations de Cryptophlebia leucotreta Meyrick (Lepidoptera: tortricidae) et de Pectinophora gossypiella Saunders (Lepidoptera : gelechiidae) en culture cotonnière en Côte d'Ivoire : de la sensibilité aux mécanismes. Thèse unique. Université Felix Houphouët Boigny Cocody. Côte d'Ivoire. 178 p.

6. Doffou, N.M., Ochou, O.G. \& Kouassi, K.P. (2011). Susceptibility of Pectinophora gossypiella (Lepidoptera : Gelechiidae) and Cryptophlebia leucotreta (Lepidoptera : Tortricidae) to insecticides used on cotton crops in Côte d'Ivoire, West Africa. Implications in insecticide resistance pest management strategies. Resist. Pest Manag., 20(2): 10-15.

7. Gutierrez, A.P., D’Oultremont, T., Ellis, C.K. \& Ponti, L. (2006). Climatic limits of pink bollworm in Arizona and California: effects of climate warming. acta oecologica 30 (2006): 353-364. DOI:10.1016/j.actao.2006.06.003

8. Hala, N., Ochou, G. O., Foua Bi, K., Allou, K., Ouraga, Y. \& Kouassi, P.(2006). Dynamique Spatio-Temporelle Des Populations D'altises, Podagrica Spp. (Coleoptera : Chrysomelidae) : Implications Agronomiques En Zones Cotonnieres De Côte D'ivoire. Agronomie Africaine 18 (1) : 41-57.

9. Lykouressis, D., Perdikis, D., Samartzis, D., Fantinou, A. \& Toutouzas, S. (2005). Management of the pink bollworm Pectinophora gossypiella (Saunders) (Lepidoptera: Gelechiidae) by 
mating disruption in cotton fields. Crop Protection 24 (2005) 177-183. DOI:10.1016/j.cropro.2004.07.007

10. Martin, T., Ochou, O.G., Hala, N.F., Vassal, J-M. \& Vaissayre, M. (2000). Pyrethroid resistance in the cotton bollworm, Helicoverpa armigera (Hübner), in West Africa. Pest. Manag. Sci. 56 : 549-554.

11. Ochou, O.G. \& Martin, T. (2002). Pyrethroïd Resistance in Helicoverpa armigera (Hübner): Recent Developments and Prospects for its Management in Côte d'Ivoire West Africa. Resist. Pest. Manag, $12(1): 10-16$.

12. Stoll, G. (2002). Protection Naturelle des Végétaux en Zones Tropicales. Vers une Dynamique de l'Information. CTA : Margraf Verlag, Allemagne ; 386p.

13. Unlu, L. \& Bilgic, A. (2004). The effects of the infestation ratio of spiny bollworm (Earias insulana) and pink bollworm (Pectinophora gossypiella) on cotton yield grown in semi-arid region of Turkey.J. Appl. Ecol, 128: 652-65.

14. Vaissayre, M. \& Cauquil, J. (2000). Principaux ravageurs et maladies du cotonnier en Afrique au Sud du Sahara. CIRAD-CTA, 60p. 